\title{
Fractional fermion charges induced by axial-vector and vector gauge potentials and parity anomaly in planar graphenelike structures
}

\author{
Angel E. Obispo* and Marcelo Hott ${ }^{\dagger}$ \\ UNESP Universidade Estadual Paulista - Campus de Guaratinguetá - DFQ. 12516-410, Guaratinguetá-SP, Brazil
}

(Received 31 May 2012; revised manuscript received 19 March 2014; published 7 April 2014)

\begin{abstract}
We show that fermion charge fractionalization can take place in a recently proposed chiral gauge model for graphene even in the absence of Kekule distortion in the graphene honeycomb lattice. In this model, electrons couple in a chiral way to a pseudomagnetic field with a vortex profile in such a way that it can be used to describe the influences of topological defects, such as disclinations, on the electronic states. We also extend the model by adding the coupling of fermions to an external magnetic field and show that the fermion charge can be fractionalized by means of only gauge potentials. It is shown that the chiral fermion charge can also have fractional value. We also relate the fractionalization of the fermion charge to the parity anomaly in an extended quantum electrodynamics, which involves vector and axial-vector gauge fields.
\end{abstract}

\section{INTRODUCTION}

It is known that the single-particle spectrum for the charge carriers on a honeycomb lattice described by means of a tight-binding Hamiltonian contains two zero-energy Dirac points. The tight-binding Hamiltonian, taken in the continuum limit and linearized around the Dirac points, reveals that the kinetics of the charge carriers is governed by a single free Dirac equation for a two-flavor spinor whose components designate massless fermions on the two triangular sublattices of the honeycomb lattice. A convenient coupling of the Fermi surface charge carriers to distortions of the lattice, which preserves the invariance of the system under time and space reversion transformations, opens a mass gap in the single-particle energy dispersion relation. Hou, Chamon, and Mudry [1] have shown that when such lattice distortions exhibit a vortexlike profile, there are zero modes excitations (midgap states) in the singleparticle energy spectrum. The vorticity of the distortion, either $n \geqslant 1$ or $n \leqslant-1$, determines which one of the sublattices supports $|n|$ zero modes. From the existence of fermion zero modes and from the sublattice symmetry, they show that the fermion quantum charge is fractionalized, similarly to what was shown to occur in one dimensional systems [2], such as polyacetylene [3].

Jackiw and $\mathrm{Pi}$ [4] have proposed a chiral gauge theory for graphene. They show that the system considered in Ref. [1] is invariant under global chiral gauge transformation and the invariance of the system under local chiral gauge transformation $\left[\mathrm{U}_{A}(1)\right]$ is preserved if the space component of a axial-vector gauge potential coupled in a chiral manner to the fermions is added to the system. Such a naturally incorporated gauge potential is the vector gauge field which enters the phenomenological Nielsen-Olesen-Landau-Ginzburg-Abrikosov model of a complex scalar field minimally coupled to the U(1) gauge field and whose classical minimum energy solutions exhibit vortices profiles as those binding zero-energy fermions in the scenario for fermion charge fractionalization in Ref. [1]. In this way, Jackiw and Pi have specified the dynamics for

\footnotetext{
*ovasquez@feg.unesp.br

${ }^{\dagger}$ marcelo.hott@pq.cnpq.br
}

the complex scalar field, which plays the role of the distortion of the graphene lattice. Moreover, they show that sublattice symmetry is preserved and that the fermion zero modes, as well as the fermion charge fractionalization are persistent even when the self-consistent vortex solution for the gauge field is incorporated to the system.

Approximately, two decades ago, Semenoff presented a physical realization of the parity anomaly in $2+1$ spacetime dimensions also in a honeycomb lattice [5]. In this case, the lattice contains two species of atoms and could describe for example a monolayer of boron nitride compound. The difference in energy of electrons on the atoms leads to a mass term for the fermions in the effective Dirac Hamiltonian in the continuum limit. The addition of an external gauge field coupled minimally to the fermion current leads to an abnormal induced current for each one of the fermion species, since the fermion mass breaks the parity symmetry. Such abnormal current is proportional to the sign of the mass of the fermion, and it is persistent when the mass is taken to zero. Since the sign of the mass of one species of fermion is contrary to the other one, the total fermion current is null, but an odd combination of the different currents leads to a nonzero abnormal current. For each one of the fermion species in the system considered in Ref. [5], the induced charge in the presence of an external magnetic field is due to the localized fermion zero modes. The induced charge may be fractional and proportional to the magnetic flux on the plane [6]. In the case of a vortex magnetic field, one has a finite magnetic flux and the number of fermion zero modes is proportional to the magnetic flux [7].

Furthermore, a gauge field theory also may have physics realization in honeycomb structures which present mesoscopic corrugations, whose possible influences on the electronic properties have been studied and modeled [8-10]. Topological defects such as disclinations are usually used to describe such corrugations on graphene lattices (see also Ref. [11]). These topological defects comes from the substitution of a hexagon by, for example, a pentagon, and this disclination warps (strains) the graphene sheet. Disclinations are implemented at theoretical level as a vortex of a axial-vector gauge potential which couple to the charge carriers degrees of freedom in the very same way the gauge potential enters in the chiral gauge model for graphene [4]. In fact, the introduction 
of a chiral gauge field in the Dirac equation to describe frustration in fullerenes were reported much earlier [12]. More recently, it has been explicitly demonstrated [13] that in the presence of a geometrically induced axial-vector gauge field in fullerenes, a real Kekule distortion of the hopping matrix elements can correspond to a complex Higgs field with nontrivial vorticity. In this way, an explanation for the scenario of fractionally charged vortices with localized zero modes, as proposed in Ref. [4] has been provided. Ultimately, the description of corrugation as a gauge field have become an experimental reality after the observation that Landau levels can be realized in graphene due exclusively to strain Refs. $[14,15]$.

In this work, we intend to show other features exhibited by the charge carriers in the presence of vector and axial-vector gauge potentials. In particular, we show that a vortex of a pseudomagnetic gauge field by itself binds zero-energy fermion states whose spinor structure reveals that the zeroenergy states are supported on only one of the triangular sublattices, in the same way as those presented in Refs. [1,4]. The breaking of the sublattice symmetry by the fermion zero modes implies into an induced fractional fermion charge which is proportional to the flux of the pseudomagnetic field. In fact, one can see from the spinor structure of zero modes that they are eigenstates of a Dirac matrix, which anticommutes with the Dirac Hamiltonian, and that the introduction of a staggered chemical potential term proportional to that Dirac matrix breaks down such anticommutation relation, opens up a mass gap, and reveals the origin of the sign of the fractional charge. Whether the chemical potential is positive or negative implies into the contribution of positive or negative energy states to the induced charge. We also show that the fractional fermion charge in graphene is related to the parity anomaly, provided in a very similar manner to that one proposed originally in Ref. [5], that is, by means of a axial-vector gauge field.

By imposing the invariance of the Dirac Hamiltonian under the usual U(1) local gauge transformation, we extend the system by minimally coupling the fermions to a vector gauge potential. We show that a fractional fermion charge may also be induced by a vortexlike magnetic field associated to the added vector potential with and without the pseudomagnetic field associated to the original axial-vector gauge potential. We analyze the discrete symmetries of the isolated fermion zero modes and find that they are supported on both triangular sublattices. Based on that symmetry, we show that there is room to the fractionalization of the fermion (chiral) charge as well.

In the next section, we consider the Dirac Hamiltonian resulting from the linearization around the Dirac points of the tight-binding Hamiltonian for the fermions on the graphene honeycomb structure. In this case, we start with the extended Dirac Hamiltonian proposed in Ref. [4] in the absence of the complex scalar field and find the isolated zero-energy fermion states, as well as the induced fermion charge in the presence of a vortex associated to the axial-vector gauge potential. Next, we extend the system by coupling the fermions to the usual vector gauge potential and find the isolated fermion modes. By analyzing the discrete symmetries of the problem, we calculate the induced fermion charges in the presence of the a magnetic and a pseudomagnetic vortices (or solenoids). The third section is dedicated to a detailed analysis of the fermion states when the magnetic and pseudomagnetic fields are homogeneous and, from the behavior of the lowest Landau level (LLL), we illustrate the origin of the induced fermion charges. In the fourth section, we discuss the realization of parity anomaly in an extended quantum electrodynamics (QED) in $2+1$ dimensions with vector and axial-vector gauge fields. The parity anomaly is realized by the dynamically generated Chern-Simons (C-S) terms and from them we calculate the associated induced fermion currents which recover the fermion charges obtained in the previous sections. The fifth section is left to the conclusions.

\section{FERMION ZERO MODES ON VORTEXLIKE (PSEUDO)MAGNETIC FIELDS}

It has been demonstrated in Ref. [13] that the scenario of fractionally charged vortices proposed in Ref. [4] may find its realization in fullerenes with a modified topology and modulation of the hopping strength, once the modified topology leads a real-valued modulation of the hopping strength to become a complex-valued scalar field. This is an amazing and very important outcome. Now, if one is interested to investigate further consequences due to the altered topology in graphene, without taking into account the modulation of the hopping strengths, one may ask whether fractional charge might also be induced even when no modulation (distortion) of the hopping strength in the graphene honeycomb lattice is present, that is, by considering only a pseudomagnetic field (associated to the axial-vector gauge field), which would describe a local single disclination. Naturally, we have found a positive answer to this question, since the results we present here rely upon the theorem associating the number of fermion zero-modes to the magnetic flux [7] and on the proof carried out in Ref. [16] that the index of Dirac Hamiltonian in $2+1$ space-time dimensions is proportional to the magnetic flux. What we are going to do in this section is to show such results explicitly in the context of graphene. We also show that an external magnetic field can also induce a fractional charge not only by itself, but also together with a vortexlike pseudomagnetic field if the corresponding vector gauge potential, minimally coupled to the charge carrier, also bears a vortexlike profile. We show that zero modes are supported in only one sublattice when the pseudomagnetic field is dominant and, in both sublattices when the magnetic field is dominant. Moreover, we show that the chiral fermion charge (number), induced by means of magnetic fields only, may also take fractional values under appropriate conditions on the magnetic fluxes.

The field theory Hamiltonian describing the dynamics of the electrons on the graphene honeycomb structure in the presence of a complex scalar field, which play the role of the lattice distortions, together with a vector gauge potential coupled in a chiral way to the electrons is written in a simplified version as

$$
\mathcal{H}=\int d^{2} \vec{r} \Psi^{\dagger}(\vec{r})\left[-i \vec{\alpha} \cdot \vec{\nabla}-\gamma_{5} \vec{\alpha} \cdot \overrightarrow{A_{5}}+g \beta\left(\varphi^{r}-i \gamma_{5} \varphi^{i}\right)\right] \Psi(\vec{r})
$$


where $\Psi(\vec{r})$ is the four-component spinor

$$
\Psi(\vec{r})=\left(\begin{array}{l}
\psi_{+}^{b}(\vec{r}) \\
\psi_{+}^{a}(\vec{r}) \\
\psi_{-}^{a}(\vec{r}) \\
\psi_{-}^{b}(\vec{r})
\end{array}\right),
$$

and $\vec{\alpha}, \beta$, and $\gamma_{5}$ are Dirac matrices in the representation explicit below. The superscripts in the spinor components designate the triangular sublattice, $A$ or $B$, the electrons are supported on, while the subscripts stand for each one of the Dirac points (the single-particle energy spectrum obtained from the original tight-binding Hamiltonian exhibits two Dirac points). The Kekule distortion is represented by the complex field $\varphi(\vec{r})=|\varphi(\vec{r})| e^{i \chi(\vec{r})}=\varphi^{r}(\vec{r})+i \varphi^{i}(\vec{r})$ and $\overrightarrow{A_{5}}(\vec{r})$ is the axial-vector gauge potential added to make the Hamiltonian invariant under local chiral gauge transformation $\varphi \rightarrow e^{i 2 \omega(\vec{r})} \varphi, \Psi \rightarrow e^{i \gamma_{5} \omega(\vec{r})} \Psi$ [4]. The matrix structure in (1) is revealed by means of the following gamma matrices:

$$
\begin{aligned}
\beta & =\left(\begin{array}{ll}
0 & I \\
I & 0
\end{array}\right), \quad \vec{\alpha}=\beta \vec{\gamma}=\left(\begin{array}{cc}
\vec{\sigma} & 0 \\
0 & -\vec{\sigma}
\end{array}\right), \\
\alpha^{3} & =\beta \gamma^{3}=\left(\begin{array}{cc}
\sigma_{3} & 0 \\
0 & -\sigma_{3}
\end{array}\right), \\
\gamma_{5} & =-i \alpha^{1} \alpha^{2} \alpha^{3}=\left(\begin{array}{cc}
I & 0 \\
0 & -I
\end{array}\right),
\end{aligned}
$$

where $I$ is the $2 \times 2$ identity matrix and $\vec{\sigma}=\left(\sigma_{1}, \sigma_{2}\right)$ and $\sigma_{3}$ are the Pauli matrices.

Now, we consider the Hamiltonian (1) by dropping out the interaction term of the electrons with the Kekule distortion and show that if the axial-vector gauge potential has a convenient asymptotic behavior, there will be isolated fermion zero modes in the single-particle energy spectrum. It is worth mentioning that this case is equivalent, via a similarity transformation, to the one shown in Ref. [10], where the effects of multiple and isolated disclinations in graphene lattice are studied. There it is shown that the lattice local deformation may be described by an axial-vector gauge field with a vortex profile or a Dirac equation under the influence of a cosmic string. In this vein, what we are going to show next is that the corrugation in honeycomb lattice, at least at the theoretical level, may lead by itself to the fractionalization of the fermion charge.

For the sake of simplicity, we consider

$$
A_{5}^{i}=\varepsilon^{i j} \partial_{j} \mathcal{A}_{5}(r) \Rightarrow B_{5}=-\vec{\nabla}^{2} \mathcal{A}_{5}(r),
$$

where $\mathcal{A}_{5}(r)$ is a scalar function of the radial coordinate only. The time-independent Dirac equation

$$
\vec{\alpha} \cdot\left(-i \vec{\nabla}-q \gamma_{5} \overrightarrow{A_{5}}\right) \Psi(\vec{r})=E \Psi(\vec{r})
$$

can be rewritten as four equations for the spinor components (the coupling constant $q>0$ is introduced here for future convenience). Two of them, in a simple form, are

$$
\begin{aligned}
-2 i\left[\partial_{\bar{z}}-q \partial_{\bar{z}} \mathcal{A}_{5}\right] \psi_{+}^{b} & =E \psi_{+}^{a} \text { and }-2 i\left[\partial_{z}+q \partial_{z} \mathcal{A}_{5}\right] \psi_{+}^{a} \\
& =E \psi_{+}^{b},
\end{aligned}
$$

while the equations for $\psi_{-}^{b}$ and $\psi_{-}^{a}$ are obtained by taking the complex conjugate of the above equations and by making the identifications $\psi_{-}^{b}=\left(\psi_{+}^{b}\right)^{*}$ and $\psi_{-}^{a}=\left(\psi_{+}^{a}\right)^{*}$. In the above equations, we are using the notation $z=x+i y$ and $\partial_{z}=$ $\left(\partial_{x}-i \partial_{y}\right) / 2$. In such a simple notation, one can easily check that the zero-energy non-normalized eigenstates are given by

$$
\begin{array}{rlrl}
\psi_{+}^{b} & =f(z) e^{q \mathcal{A}_{5}(r)}, & \psi_{+}^{a}=h(\bar{z}) e^{-q \mathcal{A}_{5}(r)}, \\
\psi_{-}^{b}=\overline{f(z)} e^{q \mathcal{A}_{5}(r)}, & \psi_{-}^{a}=\overline{h(\bar{z})} e^{-q \mathcal{A}_{5}(r)},
\end{array}
$$

where $f(z)$ and $h(\bar{z})$ are holomorphic functions. The normalization of the eigenfunctions depends on the functional dependence of the spinor components and the behavior of $\mathcal{A}_{5}(r)$ at $r=0$ and $r \rightarrow \infty$. Either $\psi_{ \pm}^{b}(\vec{r})$ or $\psi_{ \pm}^{a}(\vec{r})$ is normalizable, thereby one obtains one of the following zero-energy eigenstates:

$$
\begin{aligned}
& \Psi_{0}(\vec{r})=\mathcal{N}\left(\begin{array}{c}
e^{+i l \theta} \\
0 \\
0 \\
e^{-i l \theta}
\end{array}\right) r^{l} e^{q \mathcal{A}_{5}(r)} \quad \text { or } \\
& \Psi_{0}(\vec{r})=\mathcal{N}\left(\begin{array}{c}
0 \\
e^{-i l \theta} \\
e^{+i l \theta} \\
0
\end{array}\right) r^{l} e^{-q \mathcal{A}_{5}(r)},
\end{aligned}
$$

where $l$ is taken to be an integer number and $\theta$ is the angular variable in cylindrical coordinates. The degeneracy of the zeroenergy level depends on the number of values $l$ can assume and it is linked to the magnetic flux according to the theorems proved in Ref. [7]. In this section, we are concerned with finite magnetic flux configurations only.

To show that a fermion charge can be induced with such pseudomagnetic field configuration due to only the zero modes, we note that those are eigenfunctions of $\alpha^{3}$ and that the Hamiltonian operator $\vec{\alpha} \cdot\left(\vec{p}-\gamma_{5} \overrightarrow{A_{5}}\right)$ anticommutes with the matrix $\alpha^{3}$, then each eigenfunction of negative energy is obtained from an eigenfunction of positive energy by $\Psi_{-|E|}(\vec{r})=\alpha^{3} \Psi_{|E|}$. In other words, the energy spectrum is symmetric around the zero-energy level whose corresponding eigenfunctions are self-conjugate. From this particle-hole symmetry (except for the zero modes), and from the fact that the zero modes contain representatives of only one of the triangular sublattices, one obtains, by following the procedures in Refs. $[6,17,18]$, that the induced fermion number is given by

$$
N=\int d^{2} \vec{r} \frac{1}{2}\left\langle 0\left|\left[\Psi^{\dagger}(\vec{r}), \Psi(\vec{r})\right]\right| 0\right\rangle=\mp \frac{q \Phi_{5}}{4 \pi},
$$

where $\Phi_{5}=\int d^{2} \vec{r} B_{5}$ is the pseudomagnetic flux and the upper (lower) sign on the right-hand side of (9) comes out when the zero modes are assigned to fermions (antifermions).

The induced fermion charge is finite provided the magnetic flux is finite and that can be achieved by means of for example a solenoid or vortex magnetic field, such that [6] $\mathcal{A}_{5}(r \rightarrow$ $\infty) \sim-\left(\Phi_{5} / 2 \pi\right) \ln r$. For $\Phi_{5}>0$ one has $\Psi_{0}^{T}(r \rightarrow \infty) \sim$ $r^{l-\frac{q \Phi_{5}}{2 \pi}}\left(e^{+i l \theta} 00 e^{-i l \theta}\right)$ and for $\Phi_{5}<0 \Psi_{0}^{T}(r \rightarrow \infty) \sim r^{l+\frac{q \Phi_{5}}{2 \pi}}$ $\left(0 e^{-i l \theta} e^{+i l \theta} 0\right)$, where we are assuming that $l<\left[\left|\frac{q \Phi_{5}}{2 \pi}\right|-1\right]$, with $[v]$ denoting the largest integer less than $\nu$. The charge is 
fractional if the pseudomagnetic flux is an integer odd number $\left(n_{o}\right)$ of quantum of flux $h / q$, where $h$ is the Planck constant, that is,

$$
Q=e \int d^{2} \vec{r} \frac{1}{2}\left\langle 0\left|\left[\Psi^{\dagger}(\vec{r}), \Psi(\vec{r})\right]\right| 0\right\rangle=\mp n_{o} \frac{e}{2},
$$

where $e$ is the unit of electric charge.

The index theorem proved in Ref. [16] was successfully applied in Ref. [13] for the case of fullerenes with Kekulé distortions (complex scalar fields), where it was shown that index $H=q \Phi_{5} / 2 \pi$. Then our result is in consonance with their results, since the induced fermion number is by definition half the number of zero modes which is the index $H$. In fact, we can say that the fermion charge (number) is proportional to the Pontryiagin index, and in the case we consider the Kekulé distortion described by a complex scalar field, the charge is proportional to the vorticity of the scalar field. When the configurations of the vector gauge potential and of the scalar field are those resulting from the minimum energy of an Abelian-Higgs model in two space dimensions, the vorticity of the scalar field is equal to $q \Phi_{5} / 2 \pi=n$.

\section{A. Minimal coupling to a vector gauge potential, zero modes, and fermion charges fractionalization}

The Hamiltonian (1) is also invariant under the usual global gauge transformation $\Psi \rightarrow e^{i \xi} \Psi$, with $\xi$ uniform and constant. By promoting such a transformation to a local one, a vector gauge potential $\vec{A}$ must be added to the kinetic term in such a way that $\vec{A} \rightarrow \vec{A}+\vec{\nabla} \xi$.

We are now concerned with the zero-energy bound states and the fractional fermion charge when such a vector gauge potential is also taken into account. This is an interesting case in the context of graphenelike lattice, since the vector gauge potential couples equally to all quasiparticles in the system, then playing the role of a true external magnetic field, in spite of its unrealistic finite-flux configuration we have chosen here to illustrate the sole contribution of zero modes to the eventual fractional fermion charge. The (pseudo)magnetic field is an internal field describing properly observed topological defects in graphene lattice. Moreover, we need not to consider Kekulé distortions, whose developed fermion zero modes may conflict with the fermion zero modes due to the magnetic field, as discussed below.

The Dirac equation when both vector gauge potentials are present is

$$
\vec{\alpha} \cdot\left(-i \vec{\nabla}-q \gamma_{5} \overrightarrow{A_{5}}-e \vec{A}\right) \Psi(\vec{r})=E \Psi(\vec{r}),
$$

specifically when both gauge potentials engender finite magnetic flux. Here, we have also explicitly written the coupling constant $e>0$.

An interesting aspect of the Hamiltonian operator in (11) is that it anticommutes with $\alpha^{3}$ and with $\alpha^{3} \gamma_{5}$. In the previous case, the possible zero-energy solutions are eingenstates of $\alpha^{3}$, while in the case $\overrightarrow{A_{5}}=0$ and $\vec{A}$ given by

$$
A^{i}=\varepsilon^{i j} \partial_{j} \mathcal{A}(r) \Rightarrow B=-\vec{\nabla}^{2} \mathcal{A}(r),
$$

the zero-energy solutions are eigenstates of $\alpha^{3} \gamma_{5}$. In this case, the spinor components satisfy the equations

$$
\begin{aligned}
-2 i\left[\partial_{z}+e \partial_{z} \mathcal{A}\right] \psi_{+}^{a} & =E \psi_{+}^{b}, \\
-2 i\left[\partial_{\bar{z}}-e \partial_{\bar{z}} \mathcal{A}\right] \psi_{+}^{b} & =E \psi_{+}^{a}, \\
2 i\left[\partial_{z}+e \partial_{z} \mathcal{A}\right] \psi_{-}^{b} & =E \psi_{-}^{a}, \\
2 i\left[\partial_{\bar{z}}-e \partial_{\bar{z}} \mathcal{A}\right] \psi_{-}^{a} & =E \psi_{-}^{b},
\end{aligned}
$$

whose eigenfunctions for $E=0$ are given by

$$
\begin{aligned}
& \Psi_{0}(\vec{r})=\mathcal{N}\left(\begin{array}{c}
e^{+i l \theta} \\
0 \\
e^{+i l \theta} \\
0
\end{array}\right) r^{l} e^{e \mathcal{A}(r)} \text { or } \\
& \Psi_{0}(\vec{r})=\mathcal{N}\left(\begin{array}{c}
0 \\
e^{-i l \theta} \\
0 \\
e^{-i l \theta}
\end{array}\right) r^{l} e^{-e \mathcal{A}(r)} .
\end{aligned}
$$

The energy spectrum is symmetric around $E=0$, the negative energy eigenstates are obtained from the positive energy ones by means of the norm preserving operation $\Psi_{-|E|}(\vec{r})=\alpha^{3} \gamma_{5} \Psi_{|E|}(\vec{r})$. Thus the induced fermion number is given as in (9) with $q \Phi_{5}$ replaced by $e \Phi$, where $\Phi=$ $\int d^{2} \vec{r} B$. By considering $\mathcal{A}(r \rightarrow \infty) \sim-(\Phi / 2 \pi) \ln r$, we have $\Psi_{0}^{T}(r \rightarrow \infty) \sim r^{l-\frac{e \Phi}{2 \pi}}\left(e^{+i l \theta} 0 e^{+i l \theta} 0\right)$ for $\Phi>0$ and $\Psi_{0}^{T}(r \rightarrow$ $\infty) \sim r^{l+\frac{e \Phi}{2 \pi}}\left(0 e^{-i l \theta} 0 e^{-i l \theta}\right)$ for $\Phi<0$. An induced fermion charge is obtained similarly to the previous case and if the magnetic flux is quantized as an integer odd number of quantum of flux $h / e$, the fermion charge is fractionalized and given by (10).

Note that fermion zero modes are supported on both sublattices, contrary to the previous case and to the case with Kekulé distortions, when the zero modes have representatives of only one of the sublattices. This fact may not tell very much on this honeycomb lattice where all the atoms are identical, but it might signal the impossibility of zero modes when a magnetic field is taken into account together with the Kekule distortions. In fact, the Hamiltonian of this latter hypothetical model would anticommute with $\alpha^{3}$, what would imply into the cancellation of the contributions of positive and negative energy states to the induced fermion charge, then the zero modes, which would be again the sole contribution to the fermion charge, might not be developed unless one considers an extremely strong magnetic field [19]. Notwithstanding, fractionalization of the fermion charge is possible in a special circumstance when both quantized magnetic fluxes acts simultaneously. Moreover, we also show next that the chiral charge can also be fractionalized.

The spinor components satisfy the following equations:

$$
\begin{aligned}
-2 i\left[\partial_{z}+\partial_{z} \mathcal{A}_{+}\right] \psi_{+}^{a} & =E \psi_{+}^{b}, \\
-2 i\left[\partial_{\bar{z}}-\partial_{\bar{z}} \mathcal{A}_{+}\right] \psi_{+}^{b} & =E \psi_{+}^{a}, \\
2 i\left[\partial_{z}+\partial_{z} \mathcal{A}_{-}\right] \psi_{-}^{b} & =E \psi_{-}^{a}, \\
2 i\left[\partial_{\bar{z}}-\partial_{\bar{z}} \mathcal{A}_{-}\right] \psi_{-}^{a} & =E \psi_{-}^{b},
\end{aligned}
$$

In the equations above, we have set $\hbar=v_{F}=1, \mathcal{A}_{ \pm}=e \mathcal{A} \pm$ $q \mathcal{A}_{5}$ and, for sake of simplicity, we consider the following 
asymptotic behaviors $\mathcal{A}_{5}(r \rightarrow \infty) \sim-\frac{n_{5}}{q} \ln r$ and $\mathcal{A}(r \rightarrow$ $\infty) \sim-\frac{n}{e} \ln r$, with $n_{5}$ and $n$ positive integer numbers.

Normalized zero-energy states are obtained in some particular cases, namely,

$$
\begin{aligned}
\Psi_{0, l_{+}, l_{-}}^{n>n_{5}}(\vec{r}) & =\left(\begin{array}{c}
\frac{1}{\sqrt{4 \pi \int_{0}^{\infty} r^{2 l_{+}+1} e^{2 \mathcal{A}_{+}\left(r^{\prime}\right)} d r^{\prime}}} r^{l_{+}} e^{i l_{+} \theta} e^{\mathcal{A}_{+}(r)} \\
\frac{1}{\sqrt{4 \pi \int_{0}^{\infty} r^{\prime 2 l_{+}+1} e^{2 \mathcal{A}_{-}\left(r^{\prime}\right)} d r^{\prime}}} \\
0
\end{array}\right) \\
n & r^{l_{-}} e^{i l_{-} \theta} e^{\mathcal{A}_{-}(r)} \\
l_{ \pm} & =0,1, \ldots,\left[n \pm n_{5}-1\right]
\end{aligned}
$$

or

$$
\begin{aligned}
\Psi_{0, l_{+}, l_{-}}^{n<n_{5}}(\vec{r}) & =\left(\begin{array}{c}
\frac{1}{\sqrt{4 \pi \int_{0}^{\infty} r^{2 l_{+}+1} e^{2 \mathcal{A}_{+}\left(r^{\prime}\right)} d r^{\prime}}} r^{l_{+}} e^{i l_{+} \theta} e^{\mathcal{A}_{+}(r)} \\
0 \\
0 \\
\frac{1}{\sqrt{4 \pi \int_{0}^{\infty} r^{2 l_{+}+1} e^{-2 \mathcal{A}_{-}\left(r^{\prime}\right)} d r^{\prime}}} r^{l_{-}} e^{-i l_{-} \theta} e^{-\mathcal{A}_{-}(r)}
\end{array}\right), \\
n & <n_{5}, \\
l_{ \pm} & =0,1, \ldots,\left[n_{5} \pm n-1\right] .
\end{aligned}
$$

Note that $\alpha^{3} \gamma_{5} \Psi_{0, l_{+}, l_{-}}^{n>n_{5}}(\vec{r})=\Psi_{0, l_{+}, l_{-}}^{n>n_{5}}(\vec{r})$ and $\alpha^{3} \Psi_{0, l_{+}, l_{-}}^{n<n_{5}}(\vec{r})=$ $\Psi_{0, l_{+}, l_{-}}^{n<n_{5}}(\vec{r})$. On the other hand, when the magnetic fluxes are both negative, the zero modes are $\alpha^{3} \gamma_{5} \Psi_{0, l_{+}, l_{-}}^{|n|>\left|n_{5}\right|}(\vec{r})=$ $-\Psi_{0, l_{+}, l_{-} \mid}^{|n|>\left|n_{5}\right|}(\vec{r})$ and $\alpha^{3} \Psi_{0, l_{+}, l_{-}}^{|n|<n_{5} \mid}(\vec{r})=\Psi_{0, l_{+}, l_{-}}^{|n|<\left|n_{5}\right|}(\vec{r})$.

Both kinds of zero modes given by expressions (16) and (17) lead to induced fermion numbers, which depend on the net magnetic fluxes as

$$
N=\int d^{2} \vec{r} \frac{1}{2}\left\langle 0\left|\left[\Psi^{\dagger}(\vec{r}), \Psi(\vec{r})\right]\right| 0\right\rangle=\mp \frac{n}{2}, \text { for }|n|>\left|n_{5}\right|
$$

and

$$
N=\int d^{2} \vec{r} \frac{1}{2}\left\langle 0\left|\left[\Psi^{\dagger}(\vec{r}), \Psi(\vec{r})\right]\right| 0\right\rangle=\mp \frac{n_{5}}{2}, \text { for }|n|<\left|n_{5}\right| .
$$

Again, the upper (lower) sign on the right-hand side of (18) and (19) depends on whether the zero-energy states are taken as fermions (antifermions).

We have also found that a fractional (chiral) fermion number can also be induced, namely,

$$
N_{5}=\int d^{2} \vec{r} \frac{1}{2}\left\langle 0\left|\left[\Psi^{\dagger}(\vec{r}), \gamma_{5} \Psi(\vec{r})\right]\right| 0\right\rangle=\mp \frac{n_{5}}{2}, \text { for }|n|>\left|n_{5}\right|
$$

and

$N_{5}=\int d^{2} \vec{r} \frac{1}{2}\left\langle 0\left|\left[\Psi^{\dagger}(\vec{r}), \gamma_{5} \Psi(\vec{r})\right]\right| 0\right\rangle=\mp \frac{n}{2}, \quad$ for $|n|<\left|n_{5}\right|$,

if $|n|$ and $\left|n_{5}\right|$ are odd numbers.

This (chiral) fermion number is proportional to the nonvanishing fractional (anomalous) fermion charge found previously in the boron-nitride honeycomb structure [5] where two species of electrons that belong to the two different atoms implies into a mass term. Such a mass term, in the representation we have been working here, is equivalent to a staggered chemical potential. In the next section, we analyze in detail the single-particle states for electrons in the honeycomb structure and in the presence of homogeneous magnetic fields when a staggered chemical potential and a parity-breaking mass term are added to the Hamiltonian.

Last but not least, we recall that the zero-energy eigenstates (8), (14), (16), and (17) are also eigenstates of either $\alpha^{3}$ or $\alpha^{3} \gamma_{5}$ with eigenvalues \pm 1 . Weinberg has shown [16] that the index $H \doteq n_{+}-n_{-}$is proportional to the net finite magnetic flux when only vector gauge fields are present, where $n_{+}\left(n_{-}\right)$is the number of zero-energy eigenstates of a norm-preserving operator that anticommutes with $H$ corresponding to eigenvalues $+1(-1)$. In Ref. [13], the Kekulé distortion-Higgs field-is considered, $\alpha^{3}$ is the only operator that anticommutes with $H$, and at the end of the calculations index $H$ is the vorticity of the complex field.

\section{LANDAU LEVELS AND INDUCED CHARGES ON UNIFORM MAGNETIC FIELDS}

In this section, we obtain the fermion bound states when the electrons on the honeycomb structure are under the action of axial-vector and vector gauge potentials for homogeneous magnetic fields. In this analysis, we introduce terms in the Hamiltonian density, which provide gaps in the single-particle energy spectrum. Such terms are a staggered chemical potential $\mu \alpha^{3}$ and a parity-breaking "mass term" $m_{\tau} \alpha^{3} \gamma_{5}=m_{\tau} \beta \tau$. The staggered chemical potential was already considered in Refs. [18] and [20] to show the realization of irrational fermion charge induced by scalar fields and still maintaining the time-reversal symmetry. The parity-breaking mass matrix $m_{\tau} \beta \tau$ in this version of the graphene honeycomb structure is the continuous limit of the Haldane mass (energy) [21] linearized around the Dirac points [22]. We consider it as constant as in Ref. [23], but the same term was taken to be position-dependent (a domain wall) in Ref. [24] to investigate how the electronic properties of graphene are modified under such a domain wall.

When such terms are added, the resulting Hamiltonian operator anticommutes neither with $\alpha^{3}$ nor with $\alpha^{3} \gamma_{5}$ and the system does not admit any norm-preserving (conjugation) symmetry, that is, normalizable negative-energy eigenstates are no longer obtained from the normalizable positive-energy eigenstates and the lowest energy states are not necessarily self-conjugate under the operations of either $\alpha^{3}$ or $\alpha^{3} \gamma_{5}$. Nevertheless, a nonunitary operator that conjugates negativeto positive-energy eigenstates and vice versa might be found, but due to the lack of unitarity, the density of positive-energy states is different from the density of negative-energy states belonging to the continuous energy spectrum and, as a consequence, an irrational fermion number is induced [17]. Since we do not know exactly the fermion single-particle energy states in a vortexlike magnetic field, we are going to consider the case of homogeneous magnetic fields whose single-particle energy levels are the familiar Landau levels. Once the energy spectrum is discrete (no scattering states), there is no way to realize irrational fermion number. Here, the fermion number density is proportional to the surface 
density of states of the LLL whose associated eigenstates are eigenfunctions of either $\alpha^{3}$ or $\alpha^{3} \gamma_{5}$, depending on the magnitude of the applied magnetic fields.

It is important to emphasize that we do not consider the persistence of zero modes provided by Kekule distortions in the presence of a magnetic field, as was done in Refs. [19] and [25]. The results found there reveal that the sublattice symmetry is broken even in the presence of a magnetic field and that the vorticity of the Higgs field-either $n \geqslant 1$ or $n \leqslant-1$-which plays the role of a complex-valued distortion, determines which one of the sublattices supports $|n|$ zero modes, while in our results, the sublattice symmetry is broken, at the level of the lowest-energy eigenfunctions, only when the pseudomagnetic field is stronger than the regular magnetic field. In this way, the spinor structure of the zero modes will depend on which of them, between the magnetic and pseudomagnetic fields, is stronger than the other. Notwithstanding, our results could be compared with those found in Sec. V of Ref. [19], where the effects of a magnetic field, stronger than the uniform scalar fields, are analyzed. [Parenthetically, we notice that in the representantion of $\gamma$ matrices we are using, those scalar fields are equivalent to Kekulé distortions in Hamiltonian (1).] In summary, one could say that, once the spectrum is discrete, the zero modes are the ones responsible for the vacuum induced charge, which is proportional to the magnetic flux, and then is no longer fractional since the magnetic flux is infinity for uniform fields. Although this seems to be an obvious result, we present a detailed analysis on the spinor structure of all bound states and show how the zero-energy states arise by taking different limits of masses to zero. Our results are not only in consonance with those presented in the previous section, but also corroborate them. Here, we have normalizable eigenfunctions, while the normalization constants of the zeroenergy eigenstates (8), (14), (16), and (17) are ill-defined because of the singularity of the gauge vector at the origin. Moreover, our results may also be important in condensed matter systems where one or both of those parity-symmetrybreaking mass gaps considered here are unavoidable.

Now, the Dirac equation is

$$
\left[\vec{\alpha} \cdot\left(-i \vec{\nabla}-q \gamma_{5} \overrightarrow{A_{5}}-e \vec{A}\right)+\mu \alpha_{3}+m_{\tau} \beta \tau\right] \Psi(\vec{r})=E \Psi(\vec{r}),
$$

where the axial-vector and vector gauge potentials are written, respectively, as in (4) and (12) with $\mathcal{A}(r)$ and $\mathcal{A}_{5}(r)$ given by

$$
\mathcal{A}(r)=-\frac{B r^{2}}{4} \quad \text { and } \quad \mathcal{A}_{5}(r)=-\frac{B_{5} r^{2}}{4} \text {. }
$$

The set of equations for the spinor components can be written as

$$
\begin{aligned}
-i & {\left[2 \partial_{z}-\frac{\omega_{+}}{2} \bar{z}\right] \psi_{+}^{a}=\left(E-m_{+}\right) \psi_{+}^{b}, } \\
-i & {\left[2 \partial_{\bar{z}}+\frac{\omega_{+}}{2} z\right] \psi_{+}^{b}=\left(E+m_{+}\right) \psi_{+}^{a}, } \\
& i\left[2 \partial_{z}-\frac{\omega_{-}}{2} \bar{z}\right] \psi_{-}^{b}=\left(E+m_{-}\right) \psi_{-}^{a}, \\
i & {\left[2 \partial_{\bar{z}}+\frac{\omega_{-}}{2} z\right] \psi_{-}^{a}=\left(E-m_{-}\right) \psi_{-}^{b}, }
\end{aligned}
$$

where $\omega_{ \pm}=e B \pm q B_{5}$ and $m_{ \pm}=\mu \pm m_{\tau}$. This problem is exactly solvable, but the spinor structure of the eigenstates of the Hamiltonian operator depends on whether $\mu>m_{\tau}$ or $\mu<m_{\tau}$ as well as on whether $e B>q B_{5}$ or $e B<q B_{5}$. Hereafter, we take $\mu, m_{\tau} \geqslant 0$ and $e B, q B_{5}>0$, although the cases when $e B, q B_{5}<0$ and/or $\mu, m_{\tau} \leqslant 0$ can also be analyzed straightforwardly. Even in the cases we are concerned here, there are four different situations, which can be better appreciated if examined separately.

\section{Case 1: $q B_{5}>e B$}

The energy spectrum is $\left|E_{ \pm}\right|=\sqrt{2 n\left|\omega_{ \pm}\right|+m_{ \pm}^{2}}$, with $n \in \mathbb{N}$ and the eigenstates are

$$
\begin{gathered}
\Psi_{+}^{E_{+}>0}=\frac{e^{-i\left|E_{+}\right| t}}{\sqrt{2}}\left(\begin{array}{c}
\sqrt{1+\frac{m_{+}}{\left|E_{+}\right|}} R_{n, l, \omega_{+}}(r, \theta) \\
i \sqrt{1-\frac{m_{+}}{\left|E_{+}\right|}} R_{n-1, l+1, \omega_{+}}(r, \theta) \\
0 \\
0
\end{array}\right), \\
\Psi_{-}^{E_{-}>0}=\frac{e^{-i\left|E_{-}\right| t}}{\sqrt{2}}\left(\begin{array}{c}
0 \\
0 \\
-i \sqrt{1-\frac{m_{-}}{\left|E_{-}\right|}} R_{n-1, l+1, \omega_{-}}^{*}(r, \theta) \\
\sqrt{1+\frac{m_{-}}{\left|E_{-}\right|}} R_{n, l, \omega_{-}}^{*}(r, \theta)
\end{array}\right)
\end{gathered}
$$

and

$$
\begin{gathered}
\Psi_{+}^{E_{+}<0}=\frac{e^{i\left|E_{+}\right| t}}{\sqrt{2}}\left(\begin{array}{c}
\sqrt{1-\frac{m_{+}}{\left|E_{+}\right|}} R_{n, l, \omega_{+}}(r, \theta) \\
-i \sqrt{1+\frac{m_{+}}{\left|E_{+}\right|}} R_{n-1, l+1, \omega_{+}}(r, \theta) \\
0 \\
0
\end{array}\right), \\
\Psi_{-}^{E_{-}<0}=\frac{e^{i\left|E_{-}\right| t}}{\sqrt{2}}\left(\begin{array}{c}
0 \\
0 \\
i \sqrt{1+\frac{m_{-}}{\left|E_{-}\right|}} R_{n-1, l+1, \omega_{-}}^{*}(r, \theta) \\
\sqrt{1-\frac{m_{-}}{\left|E_{-}\right|}} R_{n, l, \omega_{-}}^{*}(r, \theta)
\end{array}\right),
\end{gathered}
$$

where

$$
\begin{aligned}
R_{n, l, \omega_{ \pm}}(r, \theta)= & \sqrt{\left(\frac{\left|\omega_{ \pm}\right|}{2}\right)^{l+1} \frac{n !}{\pi(n+l) !}} e^{i l \theta} r^{l} \\
& \times e^{-\frac{\left|\omega_{ \pm}\right|}{4} r^{2}} L_{n}^{l}\left(\left|\omega_{ \pm}\right| r^{2} / 2\right), \quad l \in \mathbb{N}
\end{aligned}
$$

are the Gauss-Laguerre modes in cylindrical coordinates and expressed in terms of the associated Laguerre polynomials $L_{n}^{l}\left(\left|\omega_{ \pm}\right| r^{2} / 2\right)$. Here, $R^{*}$ stands for the conjugate to $R$ whose normalization is $\int\left|R_{n, l, \omega_{ \pm}}(r, \theta)\right|^{2} r d r d \theta=1$, and we are assuming that $R_{-1, l, \omega_{ \pm}}(r, \theta)=0$. We notice that there is another set of normalizable eigenstates for $n>0$ and $l \in \mathbb{Z}_{-}$. Since they do not affect the final results on the induced charges, we do not display them here for the sake of simplicity. 
For $n=0$ and for the case $\mu>m_{\tau}\left(m_{ \pm}>0\right)$, the lowest energy states are

$$
\begin{gathered}
\Psi_{+}^{E_{+}=m_{+}}=e^{-i m_{+} t}\left(\begin{array}{c}
R_{0, l, \omega_{+}}(r, \theta) \\
0 \\
0 \\
0
\end{array}\right), \\
\Psi_{-}^{E_{-}=m_{-}}=e^{-i m_{-} t}\left(\begin{array}{c}
0 \\
0 \\
0 \\
R_{0, l, \omega_{-}}^{*}(r, \theta)
\end{array}\right) .
\end{gathered}
$$

We notice that when $m_{\tau} \rightarrow 0$, the energy states above are degenerate such that one has only one eigenspinor with the same structure found in (17), which is normalizable even when $\mu \rightarrow 0$, that is,

$$
\Psi^{E_{0}=\mu}=\left(\begin{array}{c}
\psi_{+}^{b}(\vec{r}, t) \\
0 \\
0 \\
\psi_{-}^{b}(\vec{r}, t)
\end{array}\right)=\frac{e^{-i \mu t}}{\sqrt{2}}\left(\begin{array}{c}
R_{0, l, \omega_{+}}(r, \theta) \\
0 \\
0 \\
R_{0, l, \omega_{-}}^{*}(r, \theta)
\end{array}\right),
$$

Had we started with $m_{\tau}=0$ and $\mu<0$ the LLL would have energy $E_{0}=\mu<0$ and the corresponding eigenspinor would be

$$
\Psi^{E_{0}=-|\mu|}=\left(\begin{array}{c}
\psi_{+}^{b}(\vec{r}, t) \\
0 \\
0 \\
\psi_{-}^{b}(\vec{r}, t)
\end{array}\right)=\frac{e^{i|\mu| t}}{\sqrt{2}}\left(\begin{array}{c}
R_{0, l, \omega_{+}}(r, \theta) \\
0 \\
0 \\
R_{0, l, \omega_{-}}^{*}(r, \theta)
\end{array}\right) .
$$

Then the induced fermion numbers densities, for $\mu \rightarrow 0$, are given by

$$
\rho=\frac{1}{2}\left\langle 0\left|\left[\psi^{\dagger}, \psi\right]\right| 0\right\rangle=-\operatorname{sgn}(\mu) \frac{q B_{5}}{4 \pi}
$$

and

$$
\rho_{5}=\frac{1}{2}\left\langle 0\left|\left[\psi^{\dagger}, \gamma_{5} \psi\right]\right| 0\right\rangle=-\operatorname{sgn}(\mu) \frac{e B}{4 \pi} .
$$

The above results are compatible with those found in the previous section, (19) and (21), where the fermion numbers are proportional to the flux of the (pseudo)magnetic field. The dependence on the $\operatorname{sgn}(\mu)$ is explained by the fact that when $\mu$ is taken initially as positive, the LLL is occupied by electrons on the conduction band $(E=\mu>0)$, whereas when $\mu<0$, the LLL is occupied by electrons on the valence band. The results in (31) and (32) are only valid for $\mu \rightarrow 0$, otherwise there would be a contribution (parity invariant contribution) coming from the higher Landau levels.

Although there is no norm-preserving operator that conjugates negative to positive-energy eigenstates when $\mu \neq 0$, the energy spectrum is discrete and the eigenstates can be normalized, such that the density of states from the positive and negative energy spectrum (except for the isolated mode $E_{0}=$ $\mu$ ) is equal to each other. Then their contribution vanishes when one calculates the fermion numbers themselves and it is possible to show that the fermion numbers are proportional to the magnetic flux. In fact, the results in (31) and (32) express that the fermion numbers densities are equal to the surface density of states of the LLL (or the surface density of zero modes) and, as a consequence, the degeneracy is proportional to the magnetic flux.

We emphasize that in this case, as in Eqs. (19) and (21), the fermion charge is proportional to the flux of the pseudomagnetic field, while the fermion chiral charge is proportional to the flux of the magnetic field. This will be explored in the next section to analyze the effective action for the gauge fields in the relativistic context, where we will show that the derivative expansion approximation to the effective action leads to a crossed Chern-Simons term.

The next case we treat is well known and has been recently analyzed in Ref. [23] by resorting to another representation for the reducible gamma matrices in $2+1$ (space-time) dimensions. Although they do not consider the axial-vector gauge potential, their results are more general than ours in the sense that they take inhomogeneous magnetic fields and analyze also the behavior of the chiral condensates.

\section{Case 2: $q B_{5}<e B$}

The energy spectrum is identical to the one of the previous case and the eigenstates are expressed as

$\Psi_{+}^{E_{+}>0}=\frac{e^{-i\left|E_{+}\right| t}}{\sqrt{2}}\left(\begin{array}{c}\sqrt{1+\frac{m_{+}}{\left|E_{+}\right|}} R_{n, l, \omega_{+}}(r, \theta) \\ i \sqrt{1-\frac{m_{+}}{\left|E_{+}\right|}} R_{n-1, l+1, \omega_{+}}(r, \theta) \\ 0 \\ 0\end{array}\right)$,

$\Psi_{-}^{E_{-}>0}=\frac{e^{-i\left|E_{-}\right| t}}{\sqrt{2}}\left(\begin{array}{c}0 \\ 0 \\ \sqrt{1-\frac{m_{-}}{\left|E_{-}\right|}} R_{n, l, \omega_{-}}(r, \theta) \\ -i \sqrt{1+\frac{m_{-}}{\left|E_{-}\right|}} R_{n-1, l+1, \omega_{-}}(r, \theta)\end{array}\right)$,

$\Psi_{+}^{E_{+}<0}=\frac{e^{i\left|E_{+}\right| t}}{\sqrt{2}}\left(\begin{array}{c}\sqrt{1-\frac{m_{+}}{\left|E_{+}\right|}} R_{n, l, \omega_{+}}(r, \theta) \\ -i \sqrt{1+\frac{m_{+}}{\left|E_{+}\right|}} R_{n-1, l+1, \omega_{+}}(r, \theta) \\ 0 \\ 0\end{array}\right)$,

$\Psi_{-}^{E_{-}<0}=\frac{e^{i\left|E_{-}\right| t}}{\sqrt{2}}\left(\begin{array}{c}0 \\ 0 \\ \sqrt{1+\frac{m_{-}}{\left|E_{-}\right|}} R_{n, l, \omega_{-}}(r, \theta) \\ i \sqrt{1-\frac{m_{-}}{\left|E_{-}\right|}} R_{n-1, l+1, \omega_{-}}(r, \theta)\end{array}\right)$.

For $\mu=0$ and $m_{\tau}>0$, the eigenstate of the LLL is

$$
\Psi^{E_{0}=m_{\tau}}=\left(\begin{array}{c}
\psi_{+}^{b}(\vec{r}, t) \\
0 \\
\psi_{-}^{a}(\vec{r}, t) \\
0
\end{array}\right)=\frac{e^{-i m_{\tau} t}}{\sqrt{2}}\left(\begin{array}{c}
R_{0, l, \omega_{+}}(r, \theta) \\
0 \\
R_{0, l, \omega_{-}}(r, \theta) \\
0
\end{array}\right)
$$


and exhibits the same spinor structure as the one in (16), while for $\mu=0$ and $m_{\tau}<0$, we have

$$
\Psi^{E_{0}=-\left|m_{\tau}\right|}=\left(\begin{array}{c}
\psi_{+}^{b}(\vec{r}, t) \\
0 \\
\psi_{-}^{a}(\vec{r}, t) \\
0
\end{array}\right)=\frac{e^{i\left|m_{\tau}\right| t}}{\sqrt{2}}\left(\begin{array}{c}
R_{0, l, \omega_{+}}(r, \theta) \\
0 \\
R_{0, l, \omega_{-}}(r, \theta) \\
0
\end{array}\right) .
$$

In obtaining the induced fermion charges it is convenient to separate the contribution of the LLL from the higher LL, which also contribute in this case as well, but the last contributions vanishes when $m_{\tau} \rightarrow 0$ and one finds

$$
\begin{gathered}
\rho=\frac{1}{2}\left\langle 0\left|\left[\psi^{\dagger}, \psi\right]\right| 0\right\rangle=-\operatorname{sgn}\left(m_{\tau}\right) \frac{e B}{4 \pi}, \\
\rho_{5}=\frac{1}{2}\left\langle 0\left|\left[\psi^{\dagger}, \gamma_{5} \psi\right]\right| 0\right\rangle=-\operatorname{sgn}\left(m_{\tau}\right) \frac{q B_{5}}{4 \pi},
\end{gathered}
$$

which are consistent with (18) and (20).

The spinor structure in (36) is similar to the one found in Sec. IV of Ref. [19], where the approximate zero-energy state is found when effects of the magnetic field $B$ are stronger than the effects produced by a weak (and approximately) Kekule distortion. Here, one can note the presence of representatives of both sublattices in the LLL, while in Ref. [19], there is only one spinor component, which would be equivalent to have one representative of only one of the sublattices in the context of graphene.

\section{PARITY ANOMALY AND INDUCED FERMION CHARGES: FIELD THEORETIC CALCULATIONS}

As one knows QED in $2+1$ space-time dimensions can be enriched by adding a topological mass to the gauge field [26] and that such a Chern-Simons term can be generated by means of perturbation calculation [27] and by resorting to derivative expansion [28]. The C-S term comes from the first-order in external momentum contribution of the vacuum polarization diagram when the massive fermions are set in the irreducible representation of the gamma matrices. In fact, the fermion mass breaks the parity symmetry and that is reflected in dynamical generation of the C-S term even when the mass is taken to zero, that is, the realization of parity anomaly.

Here, we show that $\mathrm{C}-\mathrm{S}$ terms can also be induced in a $\mathrm{U}(1) \times \mathrm{U}_{A}(1)$ symmetric $\mathrm{QED}$, whose Lagrangian density is

$$
\mathcal{L}=\bar{\Psi}\left[\gamma^{\mu}\left(i \partial_{\mu}+e A_{\mu}+g \gamma_{5} A_{\mu}^{(5)}\right)-\mu \gamma^{3}-m_{\tau} \tau\right] \Psi .
$$

We also compute the induced fermion currents from the C-S effective action and show that the parity anomaly is really connected to the fractional fermion charge when the flux of the pseudomagnetic field is an integer odd number of quantum of flux. The results found here are in consonance to those found in the previous two sections.

The vacuum polarization diagrams we have to consider are those shown in Fig. 1 which correspond to the vacuum polarization operator

$$
\begin{aligned}
\Pi^{\mu \nu}(k)= & -i \int \frac{d^{3} p}{(2 \pi)^{3}} \operatorname{tr}\left[\frac{1}{(\not p+\not k)-\mu \gamma^{3}-m_{\tau} \tau} \gamma^{\mu}\right. \\
& \left.\times\left(e+q \gamma^{5}\right) \frac{1}{\not p-\mu \gamma^{3}-m_{\tau} \tau} \gamma^{\nu}\left(e+q \gamma^{5}\right)\right],
\end{aligned}
$$

where $S_{F}(p)=\left(\not p-\mu \gamma_{3}-m_{\tau} \tau\right)^{-1}$ is the fermion propagator given in terms of momenta and under a staggered chemical potential and parity-breaking mass.

Here, as in the previous section, we are going to consider the contributions of the staggered chemical potential and of the parity-breaking mass separately. This is not only because we want to see the dependence of the fermion currents on the signs of $\mu$ and $m_{\tau}$ as in the previous section, but also because the fermion propagator can be easily handled when the approximations

$$
S_{F}(p) \approx S_{F, \mu}(p)=\frac{1}{\not p-\mu \gamma^{3}}\left(\mu \gg m_{\tau}\right)
$$

and

$$
S_{F}(p) \approx S_{F, m_{\tau}}(p)=\frac{1}{\not p-m_{\tau} \tau}\left(\mu<<m_{\tau}\right)
$$

are employed.

In both cases, we look for the term which is first-order in the external momentum, namely $\Pi_{1}^{\mu \nu} \sim \varepsilon^{\mu \nu \alpha} k_{\alpha}$. By taking the first approximation for the fermion propagator, Eq. (41), we note that the third diagram in Fig. 1 is the only one which gives a contribution to the $\mathrm{C}-\mathrm{S}$ terms. This can be appreciated through the following expression for $\Pi^{\mu \nu}$ :

$$
\begin{aligned}
\Pi^{\mu v}(k)= & -i \int \frac{d^{3} p}{(2 \pi)^{3}} \operatorname{tr}\left[\frac{(\not p+\not k)+\mu \gamma^{3}}{(p+k)^{2}-\mu^{2}} \gamma^{\mu}\right. \\
& \left.\times\left(e+q \gamma^{5}\right) \frac{\not p+\mu \gamma^{3}}{p^{2}-\mu^{2}} \gamma^{v}\left(e+q \gamma^{5}\right)\right],
\end{aligned}
$$

and by noting that the Levi-Civita tensor comes from $\operatorname{tr}\left(\gamma^{\mu} \gamma^{\nu} \gamma^{\alpha} \tau\right)=-4 \mathrm{i} \varepsilon^{\mu \nu \alpha}$. By isolating the relevant terms to

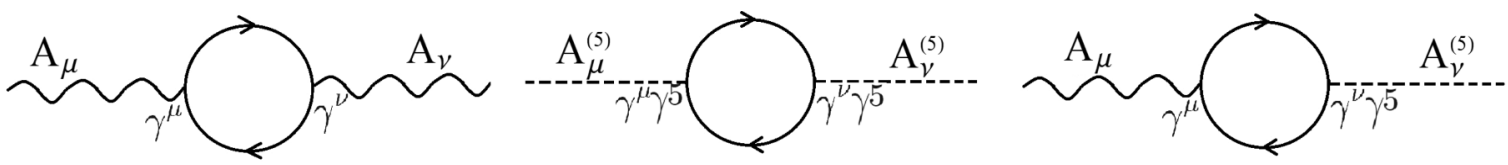

FIG. 1. The three vacuum polarization diagrams from the Lagrangian density (39). 
the $\mathrm{C}-\mathrm{S}$ contribution, one finds

$$
\begin{aligned}
\Pi_{1}^{\mu \nu} & =-\frac{\mu e q}{2 \pi^{3}} \varepsilon^{\mu \nu \alpha} k_{\alpha} \lim _{k \rightarrow 0} \int d^{3} p \frac{1}{\left(p^{2}-\mu^{2}\right)} \frac{1}{(p+k)^{2}-\mu^{2}} \\
& =-\frac{\mu e q}{2 \pi^{3}} \varepsilon^{\mu \nu \alpha} k_{\alpha} \lim _{k \rightarrow 0} \int_{0}^{1} d z \int d^{3} p \frac{1}{\left[p^{2}-\mu^{2}+k^{2} z(1-z)\right]^{2}} \\
& =i \frac{\mu e q}{2 \pi} \varepsilon^{\mu \nu \alpha} k_{\alpha} \lim _{k \rightarrow 0} \int_{0}^{1} \frac{d z}{\left[-k^{2} z^{2}+k^{2} z-\mu^{2}\right]^{1 / 2}}=i \frac{\mu}{|\mu|} \frac{e q}{2 \pi} \varepsilon^{\mu \nu \alpha} k_{\alpha} .
\end{aligned}
$$

From the effective action up to second order on the gauge fields due to the third diagram,

$$
\begin{aligned}
S_{\mathrm{eff}}^{(2)}= & \frac{1}{2} \int d^{3} x\left\{A_{\mu}(x) \Pi^{\mu v}\left(k_{\alpha}=i \partial_{\alpha}\right) A_{\nu}^{(5)}(x)\right. \\
& \left.+A_{\mu}^{(5)}(x) \Pi^{\mu \nu}\left(k_{\alpha}=i \partial_{\alpha}\right) A_{\nu}(x)\right\},
\end{aligned}
$$

one obtains a crossed C-S term, namely,

$$
\begin{aligned}
S_{\text {eff }}^{(2,1)}= & -\frac{e q}{4 \pi} \frac{\mu}{|\mu|} \varepsilon^{\mu \nu \alpha} \int d^{3} x\left\{A_{\mu}(x) \partial_{\alpha} A_{\nu}^{(5)}(x)\right. \\
& \left.+A_{\mu}^{(5)}(x) \partial_{\alpha} A_{\nu}(x)\right\} .
\end{aligned}
$$

The induced fermion current density is obtained from this contribution to the effective action through

$$
\begin{aligned}
\left\langle j^{\mu}\right\rangle & =\frac{\delta S_{e f}^{(2,1)}}{\delta A_{\mu}}=-\frac{e q}{4 \pi} \frac{\mu}{|\mu|} \varepsilon^{\mu \nu \alpha} \partial_{\alpha} A_{v}^{(5)} \\
& =-\frac{e q}{4 \pi} \frac{\mu}{|\mu|} F_{(5)}^{\mu},
\end{aligned}
$$

where $F_{(5)}^{\mu}=(-1 / 2) \varepsilon^{\mu \nu \alpha} F_{\nu \alpha(5)}$ is the dual of the (axial) field strength, whereas the (axial) fermion current density can be obtained as

$$
\begin{aligned}
\left\langle j_{5}^{\mu}\right\rangle & =\frac{\delta S_{e f}^{(2,1)}}{\delta A_{\mu}^{(5)}}=-\frac{e q}{4 \pi} \frac{\mu}{|\mu|} \varepsilon^{\mu \nu \alpha} \partial_{\alpha} A_{\nu} \\
& =-\frac{e q}{4 \pi} \frac{\mu}{|\mu|} F^{\mu} .
\end{aligned}
$$

By taking the time component of (47) and (48) one recovers the results for the fermion charge densities, Eqs. (31) and (32), respectively.

Now, we consider the effective C-S action coming from the contribution of the first two diagrams. We notice that such contributions are obtained by taking the second approximate fermion propagator in (42). Here, we calculate both contributions together, that is,

$$
\begin{aligned}
\tilde{\Pi}^{\mu \nu}(k)= & -i \int \frac{d^{3} p}{(2 \pi)^{3}} \operatorname{tr}\left[\frac{(\not p+\not k)+m_{\tau} \tau}{(p+k)^{2}-m_{\tau}^{2}} \gamma^{\mu}\right. \\
& \left.\times\left(e+q \gamma^{5}\right) \frac{\not p+m_{\tau} \tau}{p^{2}-m_{\tau}^{2}} \gamma^{\nu}\left(e+q \gamma^{5}\right)\right] .
\end{aligned}
$$

This time there will be no crossed C-S terms because $\operatorname{tr}\left(\gamma^{\mu} \gamma^{\nu} \gamma^{\alpha} \tau\right)=-4 \mathrm{i} \varepsilon^{\mu \nu \alpha}$ is obtained from the contributions proportional to $m_{\tau}$ and to $e^{2}$ and $q^{2}$, thus one has

$\tilde{\Pi}_{1,1 s t}^{\mu \nu}=i \frac{m_{\tau}}{\left|m_{\tau}\right|} \frac{e^{2}}{2 \pi} \varepsilon^{\mu \nu \alpha} k_{\alpha} \quad$ and $\quad \tilde{\Pi}_{1,2 n d}^{\mu \nu}=i \frac{m_{\tau}}{\left|m_{\tau}\right|} \frac{q^{2}}{2 \pi} \varepsilon^{\mu \nu \alpha} k_{\alpha}$, for the first and second diagrams, respectively, and the corresponding C-S effective action is

$$
\begin{aligned}
\tilde{S}_{\mathrm{eff}}^{(2,1)}= & -\frac{1}{4 \pi} \frac{m_{\tau}}{\left|m_{\tau}\right|} \varepsilon^{\mu \nu \alpha} \int d^{3} x\left\{e^{2} A_{\mu}(x) \partial_{\alpha} A_{\nu}(x)\right. \\
& \left.+q^{2} A_{\mu}^{(5)}(x) \partial_{\alpha} A_{\nu}^{(5)}(x)\right\} .
\end{aligned}
$$

From (51), one obtains the induced fermion currents

$$
\begin{aligned}
& \left\langle\tilde{j}^{\mu}\right\rangle=\frac{\delta \tilde{S}_{\mathrm{eff}}^{(2,1)}}{\delta A_{\mu}}=-\frac{e^{2}}{4 \pi} \frac{m_{\tau}}{\left|m_{\tau}\right|} F^{\mu} \quad \text { and } \\
& \left\langle\tilde{j}_{5}^{\mu}\right\rangle=\frac{\delta \tilde{S}_{e f}^{(2,1)}}{\delta A_{\mu}^{(5)}}=-\frac{q^{2}}{4 \pi} \frac{m_{\tau}}{\left|m_{\tau}\right|} F_{(5)}^{\mu},
\end{aligned}
$$

whose time-components recovers the results (37) and (38), respectively.

Most of the results shown in this section are somehow already anticipated in Secs. V and VI of Ref. [18] and Secs. III to $\mathrm{V}$ of Ref. [29], where the effective action and fractional fermion charge for a $\mathrm{U}_{V}(1) \otimes \mathrm{U}_{A}(1)$ gauge theory for graphenelike structures with space-time dependent gapopening terms are taken into account. Our inward feeling is that our results might be obtained from their results by taking the gap-opening terms associated to the complex-valued Kekulé distortions to zero and those related to $\mu$ and $m_{\tau}$ as constants. Since such limit is subtle and one is not taking into account the effect of Kekulé distortions, we would suggest to take the limits from the very beginning of the calculations. This would partially avoid complications such as inverting the full fermion propagators in the loop of the bubble diagrams, computing more diagrams than those essentially necessary to obtain the main results, and performing derivative expansions over real and complex-valued scalar fields, which are not the focus here.

\section{CONCLUSIONS}

We have obtained induced fermion charges in a continuum chiral theory for massless planar electrons in the honeycomb structure of graphene by means of only vector gauge potentials. For some configurations of the vector gauge potentials, particularly quantized magnetic vortices, we show that the induced charges can have fractional values. In this way, we have shown the possible phenomenological realization of fermion number fractionalization by pseudomagnetic fields, which seem to be a good candidate to describe defect structures, such as corrugations, in graphene lattices.

Since isolated and localized zero-energy states together with the symmetry of sublattices are crucial for the appearance of fractional charges, we have analyzed the spinor structure of 
the zero modes as well as the spectral-symmetry conjugation of the first-quantized Hamiltonian. Moreover, in order to understand and highlight the origin of zero-energy states, that is, if they come from electrons on either valence or conduction bands and that the appearance of Landau levels in graphene lattices, as due to the interaction of electrons with topological defects have been reported, we have studied in detail the energy eigenstates of fermions in homogeneous magnetic fields by taking also into account a staggered chemical potential $(\mu)$ and a parity-breaking Haldane mass term $\left(m_{\tau}\right)$, which open a mass gap in the energy spectrum (Landau levels). By taking $\mu$, $m_{\tau} \rightarrow 0$, we calculate the induced fermion number densities and the results are in consonance with the results found when the electrons are under the influence of finite magnetic fluxes.

In pursuit of the realization of quantum anomalies in condensed matter systems, we also discuss, through field-theoretic calculations, the relation of induced fermion currents with the parity anomaly in an extended quantum electrodynamics, which involves vector and axial-vector gauge fields. Unfortunately, this relation is not established beyond doubt, since the parity anomaly is only realized if parity symmetry-breaking terms such as the staggered chemical potential and the Haldane mass are present in the physical system, and that seems not to be the case in graphene, because the staggered chemical potential is due to different binding energies of electrons to two different species of atoms (the case of boron-nitride compound) and the Haldane mass has its origin in a modulated internal magnetic field whose flux by unit cell is null [21].

\section{ACKNOWLEDGMENTS}

AEO thanks to the CAPES/CNPq-IEL Nacional-Brasil program for the scholarship. This work is also partially supported by CNPq (procs. 482043/2011-3, 306316/2012-9).
[1] C.-Y. Hou, C. Chamon, and C. Mudry, Phys. Rev. Lett. 98, 186809 (2007).

[2] R. Jackiw and C. Rebbi, Phys. Rev. D 13, 3398 (1976).

[3] W. P. Su, J. R. Schrieffer, and A. J. Heeger, Phys. Rev. Lett. 42, 1698 (1979); Phys. Rev. B 22, 2099 (1980); R. Jackiw and J. R. Scrieffer, Nucl. Phys. B 190, 253 (1981).

[4] R. Jackiw and S.-Y. Pi, Phys. Rev. Lett. 98, 266402 (2007).

[5] G. W. Semenoff, Phys. Rev. Lett. 53, 2449 (1984).

[6] R. Jackiw, Phys. Rev. D 29, 2375 (1984).

[7] Y. Aharonov and A. Casher, Phys. Rev. A 19, 2461 (1979).

[8] M. A. H. Vozmediano, M. I. Katsnelson, and F. Guinea, Phys. Rep. 496, 109 (2010).

[9] Y. A. Sitenko and N. D. Vlassi, Nucl. Phys. B 787, 241 (2007).

[10] A. Cortijo and M. A. H. Vozmediano, Nucl. Phys. B 763, 293 (2006).

[11] S. Azevedo, C. Furtado, and F. Moraes, Phys. Stat. Sol. B 207, 387 (1998).

[12] J. Gonzalez, F. Guinea, and M. A. H. Vozmediano, Phys. Rev. Lett. 69, 172 (1992); Nucl. Phys. B 406, 771 (1993).

[13] J. K. Pachos, M. Stone, and K. Temme, Phys. Rev. Lett. 100, 156806 (2008).

[14] N. Levy, S. A. Burke, K. L. Meaker, M. Panlasigui, A. Zettl, F. Guinea, A. H. Castro Neto, and M. F. Crommie, Science 329, 544 (2010).
[15] F. de Juan, A. Cortijo, M. A. H. Vozmediano, and A. Cano, Nat. Phys. 7, 810 (2011).

[16] E. J. Weinberg, Phys. Rev. D 24, 2669 (1981).

[17] A. J. Niemi and G. W. Semenoff, Phys. Rep. 135, 99 (1986).

[18] C. Chamon, C.-Y. Hou, R. Jackiw, C. Mudry, S.-Y. Pi, and G. Semenoff, Phys. Rev. B 77, 235431 (2008).

[19] I. F. Herbut, Phys. Rev. B 81, 205429 (2010).

[20] C. Chamon, C.-Y. Hou, R. Jackiw, C. Mudry, S.-Y. Pi, and A. P. Schnyder, Phys. Rev. Lett. 100, 110405 (2008).

[21] F. D. M. Haldane, Phys. Rev. Lett. 61, 2015 (1988).

[22] C.-Y. Hou, Ph.D. thesis, Boston University, 2009.

[23] A. Raya and E. Reyes, Phys. Rev. D 82, 016004 (2010).

[24] G.W. Semenoff, V. Semenoff, and F. Zhou, Phys. Rev. Lett. 101, 087204 (2008).

[25] C.-Y. Hou, C. Chamon, and C. Mudry, Phys. Rev. B 81, 075427 (2010), see Appendix B.

[26] S. Deser, R. Jackiw, and S. Templeton, Phys. Rev. Lett. 48, 975 (1982); Ann. Phys. (N.Y.) 140, 372 (1982).

[27] A. N. Redlich, Phys. Rev. D 29, 2366 (1984).

[28] I. J. R. Aitchison and C. M. Fraser, Phys. Rev. D 31, 2605 (1985); K. S. Babu, A. Das, and P. Panigrahi, ibid. 36, 3725 (1987).

[29] S. Ryu, C. Mudry, C.-Y. Hou, and C. Chamon, Phys. Rev. B 80, 205319 (2009). 